\title{
Measurement of charged particle spectra in pp collisions at CMS
}

\author{
Krisztián Krajczár*ifor the CMS Collaboration \\ Eötvös Loránd University, Budapest, Hungary \\ E-mail: krisztian.krajczar@cern.ch
}

We present the plans of the CMS collaboration to measure cross sections and differential yields of unidentified charged particles produced in inelastic proton-proton collisions. The measurements of these basic observables could also serve as an important tool for calibrating and understanding the CMS detector at start-up. The tracking of very low transverse momentum charged particles will be possible down to about $100 \mathrm{MeV} / c$, with good efficiency and negligible fake rate. The tracklet reconstruction and the pixel counting analysis methods can complement the tracking and lower this limit to about $40 \mathrm{MeV} / c$.

European Physical Society Europhysics Conference on High Energy Physics, EPS-HEP 2009, July 16 - 222009

Krakow, Poland

*Speaker.

${ }^{\dagger}$ Also at KFKI RMKI, Budapest, Hungary 


\section{Introduction}

The inclusive transverse momentum and pseudorapidity distributions of hadrons produced in inelastic proton-proton collisions at the LHC provide valuable information on both non-perturbative and perturbative aspects of high-energy hadron interactions. On the one hand, soft hadron observables, not accessible to first-principle QCD calculations, can be compared to various phenomenological models. Their tuning and calibration is a basic pre-requisite to understand and control important backgrounds which affect other physics measurements at the LHC. On the other hand, the $p_{T}$ spectrum above $5 \mathrm{GeV} / c$ can be compared to perturbative calculations at next-to-leading-order accuracy using modern parton distribution and fragmentation functions.

\section{Detector}

A detailed description of the Compact Muon Solenoid (CMS) experiment can be found elsewhere [1]. The central feature of the CMS apparatus is a superconducting solenoid, of $6 \mathrm{~m}$ internal diameter. The silicon pixel and strip tracker, the crystal electromagnetic calorimeter (ECAL) and the brass-scintillator hadronic calorimeter (HCAL) are located within the field. Muons are measured in gas chambers embedded in the iron return yoke. Besides the barrel and endcap detectors, CMS has extensive forward calorimetry.

Mid-rapidity charged particles are tracked by three layers of silicon pixel detectors, made of 66 million $100 \times 150 \mu \mathrm{m}^{2}$ pixels, followed by ten microstrip layers, with strips of pitch between 80 and $180 \mu \mathrm{m}$. The silicon pixel detector consists of three barrel layers centered on the beam- line and two endcap disks on each side of the interaction point.

The methods presented here either use the full tracking detector or only the pixel barrel layers.

\section{Experimental methods}

\subsection{Charged particle tracking}

In this analysis method both pixel and strip silicon tracker detectors are used for the reconstruction of the charged particles. The procedure presented here roughly follows the standard one $[2,3]$, but it was adapted to the needs of minimum bias tracking. A good measurement of differential and integrated yields requires tracking down to $p_{T} \approx 0.1 \mathrm{GeV} / c$, since extrapolations are model dependent, in particular in the low $p_{T}$ region.

With a modified hit triplet finding algorithm the pixel detector can be employed for the reconstruction of low $p_{T}$ charged particles [4]. The particles are restricted to originate from the vicinity of the beam-line. The acceptance of the method extends down to about $0.1,0.2$ and $0.3 \mathrm{GeV} / \mathrm{c}$ in $p_{T}$ for pions, kaons and protons, respectively. The fake rate was greatly reduced, and kept at or below the percent level, with help of information present in the geometrical shape of the pixel cluster. The measured shape in the case of pixel hits, and later the measured width in the case of strip hits, is compared to the dimensions predicted from the local direction of the trajectory. This filter helps to eliminate incompatible trajectory candidates at an early stage. The obtained pixel tracks are used for finding and fitting the primary vertex or vertices [2]. 


\subsection{Tracklet reconstruction and cluster counting}

The analysis methods presented here are largely independent and complementary to the one presented in section 3.1. The cluster counting method relies on measuring and correcting the number of clusters on a given single silicon barrel layer [5]. The tracklet method is based on pairs of clusters found on different pixel barrel layers, called tracklets [6].

Two distinct methods were used for reconstructing the position of the collision point along the beam line, $v_{z}$. The first one correlates the pixel cluster size in the $z$ direction with the $z$ position of the cluster. The vertex position is then chosen such that it is consistent with the expected proportional relation between those quantities for the largest possible number of pixel clusters. The second method is based on tracklets; each pair of clusters span a straight line that defines a $z$ value where it approaches the beam line. Close-by $z$ positions are clustered and $v_{z}$ is determined as the mean $z$ value of the most populated vertex candidate cluster. The found vertices are used to calculate the pseudorapidities of clusters.

\section{Results}

\subsection{Charged particle tracking}

In this analysis we reconstructed 2 million events produced by PYTHIA [7] with the DW6T tune. Only those $\left(\eta, p_{T}\right)$ bins were used where the geometrical acceptance was above $50 \%$, the algorithmic efficiency was above $75 \%$, the fake track rate was below $2 \%$, multiple track counting was below $1 \%$, and the feed-down correction was below $10 \%$. This limits the kinematical region to $|\eta|<2.4$ and $p_{T}>0.1 \mathrm{GeV} / c$ for charged hadrons. The invariant yields were fitted by the Tsallis function $[8,9]$.

In general, results refer to the sum of positively and negatively charged particles. Symmetric positive and negative $\eta$ bins were also added. The measured invariant yields of charged hadrons are shown in the left panel of Fig. 1, as a function of $p_{T}$ and in narrow $\eta$ bins $(0.2$ units, in the range $|\eta|<2.4$ ).

The integral of the fit function is $d N_{c h} / d \eta$, therefore giving direct access to the $p_{T}$ integrated yield. It can be obtained by integrating the fit function for $p_{T}>10 \mathrm{GeV} / c$, summing the measured differential yields for $0.1<p_{T}<10 \mathrm{GeV} / c$ and adding a correction for $p_{T}<0.1 \mathrm{GeV} / c$. The pseudorapidity distribution of charged hadrons is shown in the right panel of Fig. 1. It is flat, with a slow increase towards higher $\eta$ values.

\subsection{Tracklet reconstruction and cluster counting}

The results presented in this section were obtained using 5000 DW6T PYTHIA events. After the hit selection, hit counting and corrections in each multiplicity bin, the charged particle $d N_{c h} / d \eta$ distribution is shown on the left panel in Fig. 2. The solid histogram shows the simulated distribution, while the reconstructed distribution is plotted using different symbols for the three pixel layers, with error bars corresponding to systematic errors only (estimated to be $8 \%$ ).

In case of the tracklet reconstruction analysis the pseudorapidity distribution of charged particles is obtained from the measured number of tracklets after background subtraction, efficiency and acceptance correction and normalization to the number of selected events. The pseudorapidity 

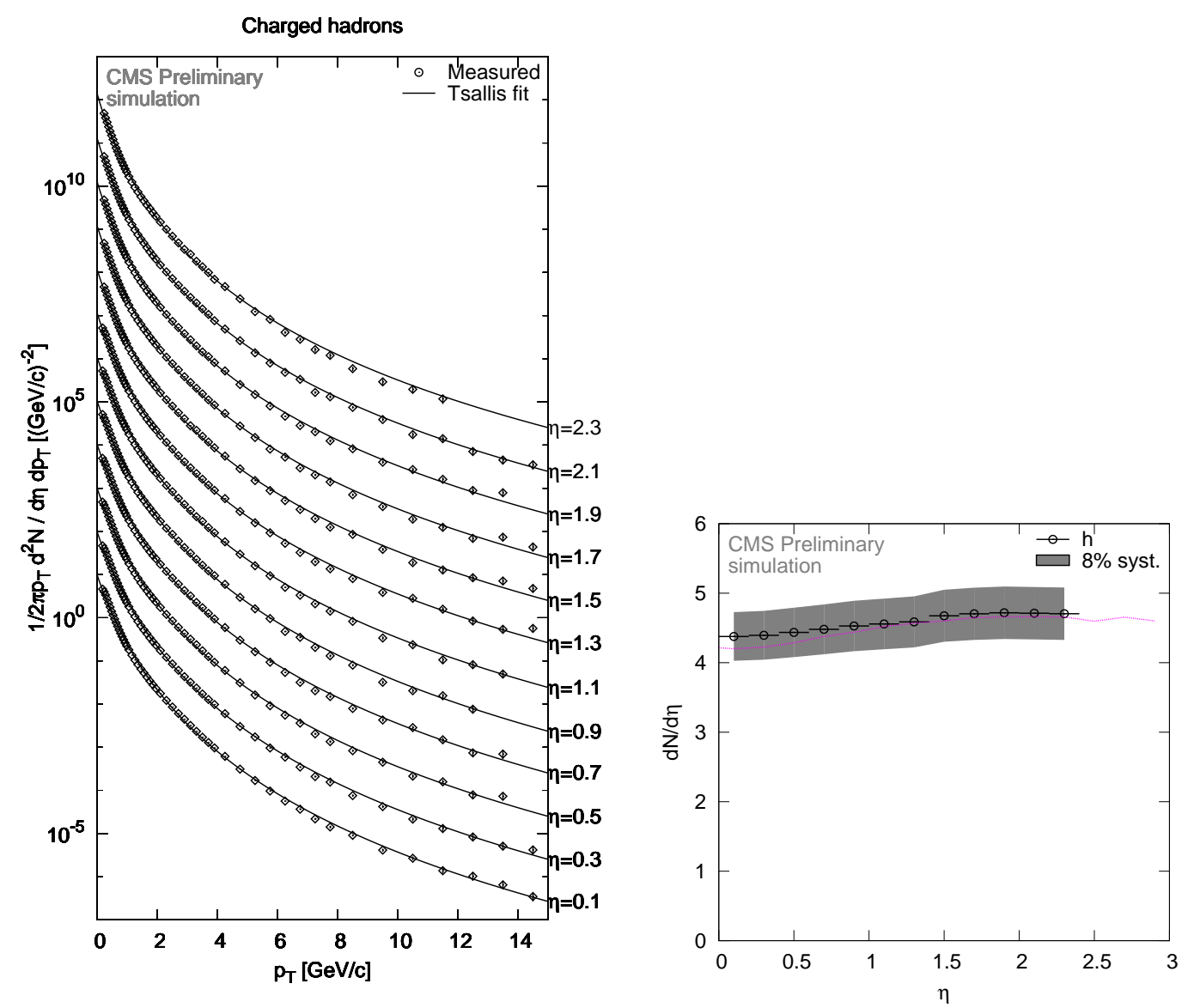

Figure 1: Left: reconstructed invariant yields of charged hadrons in the range $0<|\eta|<2.4$ in a series of 0.2 unit wide bins. Measured values (black diamonds) and empirical fit functions are plotted. The values with increasing $\eta$ are successively multiplied by 10 . Right: pseudorapidity density distribution of charged hadrons. Estimated systematic error bands (8\%) are also shown. The distribution from simulated tracks is given by the purple curve for comparison.

region within the pixel detector acceptance $(|\eta|<2.5)$ was divided into 10 bins. The $d N_{c h} / d \eta$ reconstruction is performed for each $\eta$ bin. The reconstructed $d N_{c h} / d \eta$ distributions and corresponding Monte Carlo truth distributions are presented on the right panel of Fig. 2. within statistical and systematic uncertainties. In order to check the layer by layer systematic uncertainty, we also reconstruct tracklets from first and third, as well as second and third layer hits.

\section{Summary}

Transverse momentum and pseudorapidity distributions of unidentified charged particles, produced in inelastic proton-proton collisions can be measured with good precision using the CMS tracker. The first few hours of data taking at the LHC injection energy, $0.9 \mathrm{TeV}$, and at $7 \mathrm{TeV}$ at low luminosity and event pile-up will provide the possibility to measure charged particle spectra with less than $10 \%$ systematic uncertainty, using three largely independent analysis methods. 

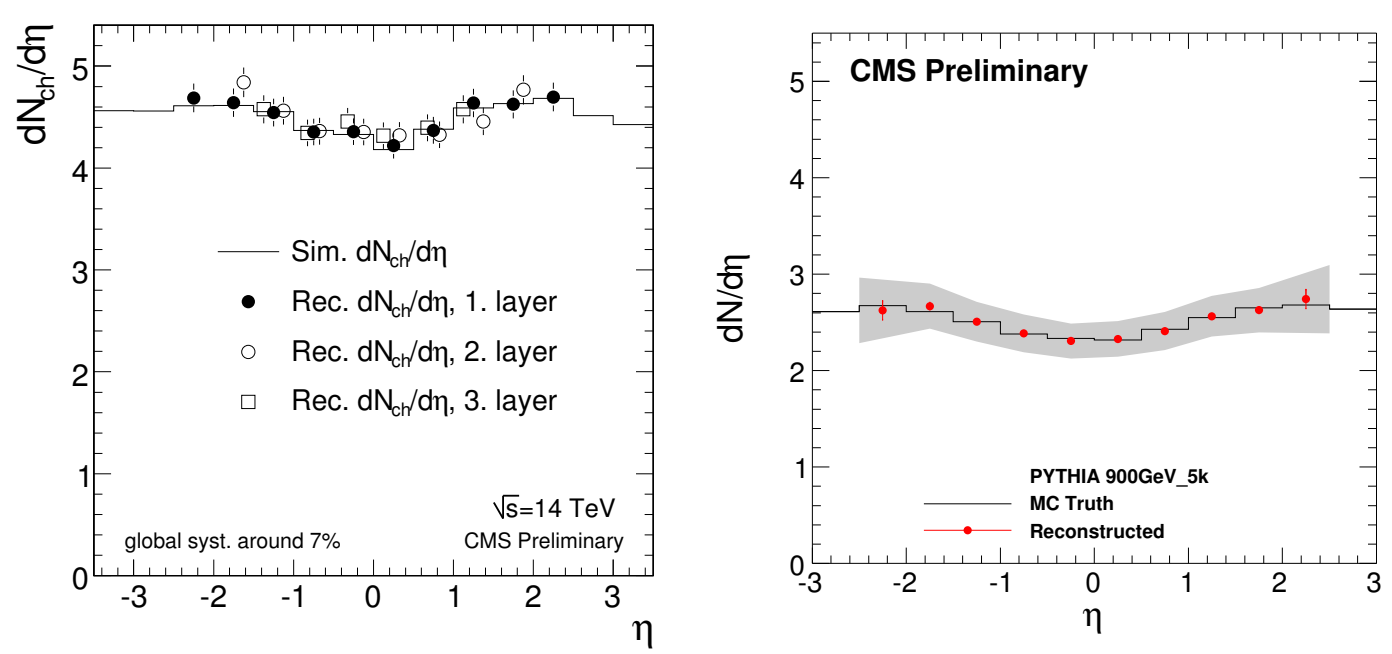

Figure 2: Left: Charged particle $d N_{c h} / d \eta$ distributions from simulated "truth" (histogram) and reconstructed (symbols) at $14 \mathrm{TeV}$. Different symbols correspond to each of the three pixel layers. Error bars show statistical errors using 5000 events. Right: Simulated and reconstructed $d N / d \eta$ distribution at $900 \mathrm{GeV}$. Error bars show statistical errors using 5000 events. The shaded area corresponds to $7.5-13.5 \%$ systematic error band.

\section{Acknowledgments}

The author wishes to thank the Hungarian Scientific Research Fund and the National Office for Research and Technology (K 48898 and NKTH-OTKA H07-C 74248) for their support.

\section{References}

[1] CMS Collaboration, R. Adolphi et al., The CMS experiment at the CERN LHC, JINST 0803 (2008) S08004.

[2] S. Cucciarelli, M. Konecki, D. Kotlinski, and T. Todorov, Track reconstruction, primary vertex finding and seed generation with the Pixel Detector, CMS Note 2006/026 (2006).

[3] W. Adam, B. Mangano, T. Speer, and T. Todorov, Track Reconstruction in the CMS tracker, CMS Note 2006/041 (2006).

[4] CMS Collaboration, Measurement of charged hadron spectra in proton-proton collisions at $\sqrt{s}=14$ TeV, CMS PAS QCD-07-001 (2007).

[5] CMS Collaboration, Pseudorapidity distributions of charged hadrons in minimum bias p-p collisions at $\sqrt{s}=14 \mathrm{TeV}, \mathrm{CMS}$ PAS QCD-08-004 (2008).

[6] CMS Collaboration, Study of Charged Hadron Multiplicity in Minimum Bias pp Collisions at $\sqrt{s}=900 \mathrm{GeV}$ and $10 \mathrm{TeV}, \mathrm{CMS}$ PAS QCD-09-002 (2009).

[7] T. Sjostrand, S. Mrenna, and P. Skands, PYTHIA 6.4 Physics and Manual, JHEP 05 (2006) 026

[8] C. Tsallis, Possible Generalization of Boltzmann-Gibbs Statistics, J. Stat. Phys. 52 (1988) 479-487

[9] T. S. Biro, G. Purcsel, G. Gyorgyi, and A. Jakovac, A non-conventional description of quark matter, J. Phys G31 (2005) S759-S763 